\title{
A VARIANT ON THE NOTION OF A DIOPHANTINE $s$-TUPLE
}

\author{
WILLIAM D. BANKS ${ }^{\dagger}$ \\ Department of Mathematics University of Missouri Columbia, MO 65211 USA \\ e-mail:bbanks@math.missouri.edu \\ FLORIAN LUCA \\ Instituto de Matemáticas Universidad Nacional Autónoma de México C.P. 58089, \\ Morelia, Michoacán, México \\ e-mail:fluca@matmor.unam.mx \\ and LÁSZLÓ SZALAY \\ Institute of Mathematics and Statistics University of West Hungary H-9400 Sopron, \\ Erzsébet u. 9, Hungary \\ e-mail: laszalay@ktk.nyme.hu
}

(Received 26 December 2007; accepted 15 July 2008)

\begin{abstract}
We show that there is an infinite set $\mathcal{S}$ of natural numbers with the property that $1+\prod_{n \in \mathcal{R}} n$ is square-free for every finite subset $\mathcal{R} \subseteq \mathcal{S}$.

2000 Mathematics Subject Classification. MSC Numbers: 11B99, 11N25.
\end{abstract}

\section{Introduction.}

1.1. Diophantine $s$-tuples. In the third century, Diophantus of Alexandria studied sets $\mathcal{S}$ of positive rational numbers with the property that $1+m n$ is the square of a rational number for all $m, n \in \mathcal{S}, m \neq n$. One example he found was the set

$$
\mathcal{S}_{d}=\left\{\frac{1}{16}, \frac{33}{16}, \frac{17}{4}, \frac{105}{16}\right\} .
$$

In the 17th century, Fermat considered Diophantus' problem, but he was mainly interested in sets that contain only natural numbers. A set of this type is called a Diophantine s-tuple if it has $s$ elements. Fermat found the first Diophantine quadruple:

$$
\mathcal{S}_{f}=\{1,3,8,120\} .
$$

Euler showed that Fermat's set can be extended to a larger set of rational numbers with Diophantus' property, namely,

$$
\mathcal{S}_{e}=\left\{1,3,8,120, \frac{777480}{8288641}\right\} .
$$

On the other hand, Baker and Davenport [1] showed that Fermat's set $\mathcal{S}_{f}$ cannot be extended to include a fifth natural number. Dujella [3] has shown that there are no

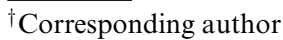


Diophantine sextuples and that there are at most finitely many Diophantine quintuples; it is unknown whether any such quintuples exist.

1.2. Generalizations. The notion of a Diophantine $s$-tuple is easily generalized by replacing the set of square numbers with an arbitrary set of natural numbers.

Definition 1. For a given set $\mathcal{A} \subseteq \mathbb{N}$, we say that $\mathcal{S} \subseteq \mathbb{N}$ is $\mathcal{A}$-Diophantine if $1+m n \in \mathcal{A}$ for all $m, n \in \mathcal{S}, m \neq n$.

With this terminology, a Diophantine $s$-tuple is simply an $\mathbb{N}^{2}$-Diophantine set with $s$ elements, where $\mathbb{N}^{2}$ is the set of square numbers. The result of Dujella asserts that $\# \mathcal{S} \leqslant 5$ for every $\mathbb{N}^{2}$-Diophantine set $\mathcal{S}$ and $\# \mathcal{S}=5$ holds for at most finitely many such sets $\mathcal{S}$.

One can also consider the following stronger condition on a set $\mathcal{S} \subseteq \mathbb{N}$ :

Definition 2. Given $\mathcal{A} \subseteq \mathbb{N}$, we say that $\mathcal{S} \subseteq \mathbb{N}$ is strongly $\mathcal{A}$-Diophantine if $1+$ $\prod_{n \in \mathcal{R}} n \in \mathcal{A}$ for every finite subset $\mathcal{R} \subseteq \mathcal{S}$.

It is easy to check that the set

$$
\mathcal{S}_{p}=\{2,3,6,26,90,336,476,3926\}
$$

has the property that $1+m n$ is a prime number for all $m, n \in \mathcal{S}_{p}, m \neq n$. In other words, $\mathcal{S}_{p}$ is $\mathcal{P}$-Diophantine, where $\mathcal{P}$ is the set of prime numbers. The set $\mathcal{S}_{p}$ is not strongly $\mathcal{P}$-Diophantine, but such sets do exist and are easily found by computer (e.g., $\left.\mathcal{S}_{p}^{*}=\{1,2,6,96\}\right)$. It is natural to ask whether there exists a $\mathcal{P}$-Diophantine set with infinitely many elements, and we conjecture that this is the case. In Section 3, we show that a well-known and widely believed conjecture of Dickson implies the existence of a strongly $\mathcal{P}$-Diophantine set of infinite cardinality.

1.3. Statement of the main result. In this note, we focus on a variant of Diophantus' problem with square-free numbers rather than square numbers. Our aim is to prove the existence of a strongly $\mathcal{A}$-Diophantine set of infinite cardinality, where $\mathcal{A}$ is the set of square-free natural numbers.

THEOREM 1. There is an infinite set $\mathcal{S} \subseteq \mathbb{N}$ with the property that $1+\prod_{n \in \mathcal{R}} n$ is square-free for every finite subset $\mathcal{R} \subseteq \mathcal{S}$. Moreover, for $x \geqslant 3$, we have

$$
\#\{n \leqslant x: n \in \mathcal{S}\} \gg \sqrt{\log \log x} .
$$

2. Construction. In what follows, the letter $p$ always denotes a prime number. For a positive integer, $\omega(n)$ denotes the number of distinct prime divisors of $n$. For positive functions $f$ and $g$, the notation $f \ll g$ means that the inequality $f \leqslant c g$ holds with some absolute constant $c>0$.

Our principal tool is the following technical lemma, which is a consequence of the more general result (Lemma 2) of Luca and Shparlinski [4]: 
LeMma 1. For any real number $y \geqslant 2$, let $K=\prod_{p \leqslant y} p$. Let $\left\{A_{1}, \ldots, A_{s}\right\}$ be a set of positive integers with the property that the products

$$
P_{\mathcal{T}}=\prod_{j \in \mathcal{T}} A_{j} \quad(\mathcal{T} \subseteq \mathcal{U}=\{1, \ldots, s\})
$$

are pairwise distinct and put

$$
F(X)=\prod_{\mathcal{T} \subseteq\{1, \ldots, s\}}\left(P_{\mathcal{T}} K X+1\right) \in \mathbb{Z}[X] .
$$

Finally, let $\Delta$ be the product of the distinct primes $p>y$ that divide the product

$$
\prod_{\substack{\mathcal{T}_{1}, \mathcal{T}_{2} \subseteq\{1, \ldots, s\} \\ \mathcal{T}_{1} \neq \mathcal{T}_{2}}}\left|P_{\mathcal{T}_{1}}-P_{\mathcal{T}_{2}}\right|
$$

Then,

$$
\#\{n \leqslant x: F(n) \text { is square-free }\} \geqslant x\left(1-\frac{2^{s}}{y}\right)^{\omega(\Delta)}-2^{s \omega(\Delta)}-\frac{2^{s} x}{y}-2^{s} \sqrt{M x},
$$

where $M=1+P_{\mathcal{U}} K$.

Proof of Theorem 1. For every real number $t$, we write $\exp _{2}(t)=\exp \left(e^{t}\right)$, and we put

$$
f(t)=\exp _{2}\left(16 t^{2}\right) \quad \text { and } \quad g(t)=\log f(t+1 / 4)=e^{16 t^{2}+8 t+1} .
$$

To prove the theorem, we construct an infinite sequence $A_{1}, A_{2}, A_{3}, \ldots$ of distinct positive integers such that for every integer $s \geqslant 1$ the following properties hold:

(i) the products $P_{\mathcal{T}}=\prod_{j \in \mathcal{T}} A_{j}$ with $\mathcal{T} \subseteq\{1, \ldots, s\}$ are pairwise distinct;

(ii) the bound $A_{j} \leqslant f(j)$ holds for each $j=1, \ldots, s$;

(iii) the number $1+P_{\mathcal{T}}$ is square-free for every subset $\mathcal{T} \subseteq\{1, \ldots, s\}$.

Assuming this has been done, we put $\mathcal{S}=\left\{A_{j}: j \geqslant 1\right\}$. Then, for every finite subset $\mathcal{R} \subseteq \mathcal{S}$, we have $1+\prod_{n \in \mathcal{R}} n=1+P_{\mathcal{T}}$, where $\mathcal{T}=\left\{j: A_{j} \in \mathcal{R}\right\}$; hence, this number is square-free. As the construction in the following text produces a set $\mathcal{S}$ with $A_{1}=2$, it suffices to establish the lower bound (1) for all sufficiently large values of $x$. For such $x$, we let $s$ be determined by the inequalities

$$
f(s)=\exp _{2}\left(16 s^{2}\right)<x \leqslant \exp _{2}\left(16(s+1)^{2}\right) .
$$

Then,

$$
\#\{n \leqslant x: n \in \mathcal{S}\} \geqslant \#\left\{A_{1}, \ldots, A_{s}\right\}=s \gg \sqrt{\log \log x}
$$

as required.

Turning now to our construction, let $A_{1}=2$, and note that $(i)-(i i i)$ hold with $s=1$. Proceeding by induction, we suppose that $A_{1}, \ldots, A_{s}$ have been defined and satisfy $(i)-(i i i)$ for some integer $s \geqslant 1$. We find a new integer $A_{s+1} \neq A_{j}$ for $j=1, \ldots, s$ such that the longer sequence $A_{1}, \ldots, A_{s+1}$ satisfies: 
(iv) the products $P_{\mathcal{T}^{\prime}}=\prod_{j \in \mathcal{T}^{\prime}} A_{j}$ with $\mathcal{T}^{\prime} \subseteq\{1, \ldots, s+1\}$ are pairwise distinct;

(v) the bound $A_{j} \leqslant f(j)$ holds for each $j=1, \ldots, s+1$;

(vi) the number $1+P_{\mathcal{T}^{\prime}}$, is square-free for every subset $\mathcal{T}^{\prime} \subseteq\{1, \ldots, s+1\}$.

To this end, we now define

$$
y=g(s) \quad \text { and } \quad K=\prod_{p \leqslant y} p .
$$

Using the upper bound $K \leqslant e^{2 y}$ (see [5, Chapter I.1.2, Theorem 4]), we have

$$
K \leqslant e^{2 g(s)} .
$$

From (ii), we derive the bound

$$
P_{\mathcal{S}}=\prod_{j=1}^{s} A_{j} \leqslant f(s)^{s} .
$$

Put $M=1+P_{\mathcal{S}} K$. Using the previous bound together with (4), we see that

$$
M \leqslant 2 P_{\mathcal{S}} K \leqslant 2 f(s)^{s} e^{2 g(s)} .
$$

Now let $\Delta$ be the product of the distinct primes $p>y$ that divide the product (2). Since

$$
\left|P_{\mathcal{T}_{1}}-P_{\mathcal{T}_{2}}\right|<P_{\mathcal{S}} \leqslant f(s)^{s}
$$

for each factor in (2), we have the crude bound

$$
\Delta \leqslant P_{\mathcal{S}}^{2^{s+1}} \leqslant f(s)^{s 2^{2+1}} .
$$

As $\Delta$ is composed of primes exceeding $y$, it follows that

$$
\omega(\Delta) \leqslant \frac{\log \Delta}{\log y} \leqslant \frac{s 2^{s+1} \log f(s)}{\log g(s)} .
$$

Let

$$
F(X)=\prod_{\mathcal{T} \subseteq\{1, \ldots, s\}}\left(P_{\mathcal{T}} K X+1\right) \in \mathbb{Z}[X] .
$$

Using Lemma 1 with $x=f(s+1 / 4)^{4}=e^{4 g(s)}$ together with the bounds (5) and (6), we deduce that

$$
\#\left\{n \leqslant e^{4 g(s)}: F(n) \text { is square-free }\right\} \geqslant L_{1}-L_{2}-L_{3}-L_{4},
$$

where

$$
\begin{aligned}
& L_{1}=e^{4 g(s)}\left(1-\frac{2^{s}}{g(s)}\right)^{s 2^{2+1} \log f(s) / \log g(s)} ; \\
& L_{2}=2^{s^{2} 2^{s+1} \log f(s) / \log g(s)}
\end{aligned}
$$




$$
\begin{aligned}
& L_{3}=\frac{2^{s} e^{4 g(s)}}{g(s)} \\
& L_{4}=2^{s} \sqrt{2 f(s)^{s} e^{6 g(s)}} .
\end{aligned}
$$

Since $\log (1-t) \geqslant-2 t$, if $0 \leqslant t \leqslant 1 / 2$, and $g(s)=e^{16 s^{2}+8 s+1} \geqslant 2^{s+1}$, we have

$$
\log \left(\frac{L_{1}}{e^{4 g(s)}}\right)=\frac{s 2^{s+1} \log f(s)}{\log g(s)} \log \left(1-\frac{2^{s}}{g(s)}\right) \geqslant-\frac{s 2^{2 s+2} \log f(s)}{g(s) \log g(s)} .
$$

In view of the definitions (3), it follows that

$$
\frac{s 2^{2 s+2} \log f(s)}{g(s) \log g(s)}=\frac{s 2^{2 s+2}}{\left(16 s^{2}+8 s+1\right) e^{8 s+1}} \leqslant 10^{-4} .
$$

Combining this bound with (8), we deduce that

$$
L_{1} \geqslant 0.8 e^{4 g(s)} \text {. }
$$

Similarly, we have

$$
\log L_{2} \leqslant \frac{s^{2} 2^{s+1} \log f(s)}{\log g(s)} \leqslant 10^{-4} \leqslant 4 g(s)-\log 5
$$

and therefore

$$
L_{2} \leqslant 0.2 e^{4 g(s)}
$$

Since $g(s) \geqslant 5 \cdot 2^{s}$, we also have

$$
L_{3} \leqslant 0.2 e^{4 g(s)}
$$

Finally, by the definitions (3), we see that

$$
\log L_{4} \leqslant s+1+0.5 s \log f(s)+3 g(s) \leqslant 4 g(s)-\log 5
$$

since

$$
e^{16 s^{2}+8 s+1} \geqslant s+1+0.5 s e^{16 s^{2}}+\log 5
$$

therefore,

$$
L_{4} \leqslant 0.2 e^{4 g(s)}
$$

Now, inserting the estimates (9)-(12) into (17), it follows that

$$
\#\left\{n \leqslant e^{4 g(s)}: F(n) \text { is square-free }\right\} \geqslant 0.2 e^{4 g(s)} \geqslant 2^{2 s}+1 .
$$

Hence, there is a positive integer $n \leqslant e^{4 g(s)}$ such that $F(n)$ is square-free, and

$$
n K \neq \frac{P_{\mathcal{T}_{1}}}{P_{\mathcal{T}_{2}}} \quad \text { for all subsets } \mathcal{T}_{1}, \mathcal{T}_{2} \text { of }\{1, \ldots, s\}
$$


Put $A_{s+1}=n K$ for any such $n$ and note that

$$
A_{s+1} \neq A_{j}=\frac{P_{\{j\}}}{P_{\varnothing}} \quad(j=1, \ldots, s) .
$$

It remains to show that the sequence $A_{1}, \ldots, A_{s+1}$ satisfies $(i v)-(v i)$. Since the products $P_{\mathcal{T}^{\prime}}=\prod_{j \in \mathcal{T}^{\prime}} A_{j}$ with $\mathcal{T}^{\prime} \subseteq\{1, \ldots, s+1\}$ all have the form $P_{\mathcal{T}}$ or $P_{\mathcal{T}} A_{s+1}$ for a subset $\mathcal{T} \subseteq\{1, \ldots, s\}$, namely, $\mathcal{T}=\mathcal{T}^{\prime} \backslash\{s+1\}$, the property (iv) is an immediate consequence of $(i)$ and (13). Taking (ii) into account, property $(v)$ is a consequence of the following bound:

$$
A_{s+1}=n K \leqslant e^{4 g(s)} e^{2 g(s)}=\exp \left(6 e^{16 s^{2}+8 s+1}\right) \leqslant \exp \left(e^{16 s^{2}+32 s+16}\right)=f(s+1) .
$$

Finally, property ( $v i$ ) follows from ( $i i i)$ and the fact that for every subset $\mathcal{T} \subseteq\{1, \ldots, s\}$, the number $1+P_{\mathcal{T}} A_{s+1}=1+P_{\mathcal{T}} K n$ is square-free since it divides the square-free number $F(n)$.

3. Remarks. Let $\mathcal{A}$ be the set of square-free natural numbers and let $\mathcal{S}$ be strongly $\mathcal{A}$-Diophantine as in Theorem 1 . It would be interesting either to improve the lower bound (1) on $\#(\mathcal{S} \cap[1, x])$ or to find a construction of such a set that yields a somewhat comparable upper bound for this quantity.

Suppose that $A_{1}<\cdots<A_{s}$ are the first $s$ elements in a strongly $\mathcal{A}$-Diophantine set $\mathcal{S}$. For a fixed subset $\mathcal{R} \subseteq\{1, \ldots, s\}$, the expectation that a random integer $n$ has the property that $n \prod_{j \in \mathcal{R}} A_{j}+1$ is square-free is $c_{\mathcal{R}} \cdot 6 / \pi^{2} \geqslant 6 / \pi^{2}$, where $c_{\mathcal{R}}=$ $\prod_{p \mid \prod_{j \in \mathcal{R}} A_{j}}\left(1-p^{-2}\right)^{-2}$. If we assume that these events are independent as $\mathcal{R}$ varies, then the probability that these numbers are simultaneously square-free for all subsets $\mathcal{R} \subseteq\{1, \ldots, s\}$ exceeds $\left(6 / \pi^{2}\right)^{2^{s}}$. Therefore, writing $x=(s+1)\left(\pi^{2} / 6\right)^{2^{s}}$, it is reasonable to expect that the interval $[1, x]$ contains at least $s+1$ numbers $n$ with this property if $s$ is large; in particular, at least one of them is not in the set $\left\{A_{1}, \ldots, A_{s}\right\}$. Since $s \sim c \log \log x$, where $c=1 / \log 2$, this heuristic argument suggests that there exists a strongly $\mathcal{A}$-Diophantine set $\mathcal{S}$ for which $\#(\mathcal{S} \cap[1, x]) \asymp \log \log x$ as $x \rightarrow \infty$.

Here we give some numerical examples. The finite set

$$
\mathcal{S}=\{1,2,5,6,9,21,42,101,330,5738,71190,206083605\}
$$

is strongly $\mathcal{A}$-Diophantine. Based on the heuristic argument, we expect that the next integer that can be added to this set, assuming it exists, must be quite large (if $A_{1}<$ $A_{2}<\cdots$ are the elements of $\mathcal{S}$, then the number of digits in the decimal representation of $A_{j}$ should grow as an exponential function of $j$ ). The set

$$
\mathcal{S}=\{1,2,5,6,9,10,14,18,21,30,33,42,45,50,64,65,77,81,82,92,100\}
$$

is $\mathcal{A}$-Diophantine (but not strongly so). This set was produced by using a greedy algorithm and can be extended to include 1, 229 numbers below $10^{8}$.

Let $\mathcal{B}$ be the set of natural numbers that are not square-free. Terr [6] has shown that for any integer $k$, there exists an infinite set $\mathcal{S}$ such that $k+m n \in \mathcal{B}$ for all $m, n \in \mathcal{S}$, $m \neq n$. In particular, there exists a $\mathcal{B}$-Diophantine set with infinitely many elements.

Since the set $\mathcal{P}$ of prime numbers is contained in the set $\mathcal{A}$ of square-free numbers, in view of Theorem 1 it is natural to ask whether there exists a $\mathcal{P}$-Diophantine set with infinitely many elements. We expect that the answer to this question is yes, but we do 
not know how to approach it. If the first $s$ elements $A_{1}<\cdots<A_{s}$ in $\mathcal{S}$ have already been constructed, then the collection of linear polynomials

$$
f_{\mathcal{R}}(X)=X \prod_{j \in \mathcal{R}} A_{j}+1 \quad(\varnothing \neq \mathcal{R} \subseteq\{1, \ldots, s\})
$$

satisfies the hypothesis of Dickson's generalized twin prime conjecture (see [2]); that is, for every prime $p$ there is an integer $n$ such that $p \nmid f_{\mathcal{R}}(n)$ for every $\mathcal{R}$ (indeed, one can take any $n$ that is divisible by $p$ ). Then, Dickson's conjecture asserts that there is an integer $A_{s+1}>A_{s}$ such that $f_{\mathcal{R}}\left(A_{s+1}\right)$ is prime for every $\mathcal{R}$ and this integer can be incorporated into the set $\mathcal{S}$.

ACKNOWLEDGEMENTS. We thank the referee for useful comments on the original manuscript. This work began during a mutual visit by the authors to the University of West Georgia for the INTEGERS Conference 2007 and continued during a subsequent visit by the third author to the University of Missouri; the support and kind hospitality of these institutions are gratefully acknowledged. During the preparation of this paper, F.L. was supported in part by Grants SEP-CONACyT 46755 and PAPIIT IN100508 and L.S. was supported in part by a János Bolyai scholarship of HAS and the Hungarian National Foundation for Scientific Research grant nos. T 048945 MAT and K 61800 FT2.

\section{REFERENCES}

1. A. Baker and H. Davenport, The equations $3 x^{2}-2=y^{2}$ and $8 x^{2}-7=z^{2}$, Quart. $J$. Math. Oxford Ser. 20(2) (1969), 129-137.

2. L. E. Dickson, A new extension of Dirichlet's theorem on prime numbers, Messenger Math. 33 (1904), 155-161.

3. A. Dujella, There are only finitely many Diophantine quintuples, J. Reine Angew. Math. 566 (2004), 183-214.

4. F. Luca and I. E. Shparlinski, Approximating positive reals by ratios of kernels of consecutive integers, in Diophantine analysis and related fields, Sem. Math. Sci. 35 (Keio Univ., Yokohama, 2006). 141-148.

5. G. Tenenbaum, Introduction to analytic and probabilistic number theory. Cambridge Studies in Advanced Mathematics, 46 (Cambridge University Press, Cambridge, 1995).

6. D. Terr, Solution of problem H-520, Fibonacci Quart. 36 (1998), 94-95. 